\title{
Heartfelt Swallow: Management of a Case of Multiple Ingested Foreign Bodies
}

\author{
Malka Davina Kirschenbaum, BS, Aditya Safaya, MD, Seungwhan Pee, MD, \\ Gustavo Stringel, MD, MBA \\ Westchester Medical Center, New York Medical College, Valhalla New York, USA \\ (all authors).
}

\begin{abstract}
Background: Sponge bezoars will absorb luminal contents, enlarge, and subsequently harden. There is significant potential for the development of intestinal obstruction and perforation.

Methods: We report the case of an unsuspected polyurethane foam (PUF) sponge bezoar causing intestinal obstruction in an 8-year-old boy undergoing laparoscopy for an ingested lithium battery that was adherent to the cecum or the appendix.

Results: The PUF sponge bezoar was removed from the small intestine via laparoscopic-assisted enterotomy. The battery was removed from the colon via colonoscopy after multiple attempts failed to retrieve it during surgery and after surgery with laxatives.

Conclusion: This case raises awareness about the presence of unsuspected multiple ingested foreign bodies causing complications and the benefits of laparoscopy in identifying them and facilitating safe retrieval.
\end{abstract}

Key Words: Bezoar intestinal obstruction, Intestinal foreign body, Polyurethane foam, Sponge ingestion, Swallowed batteries.

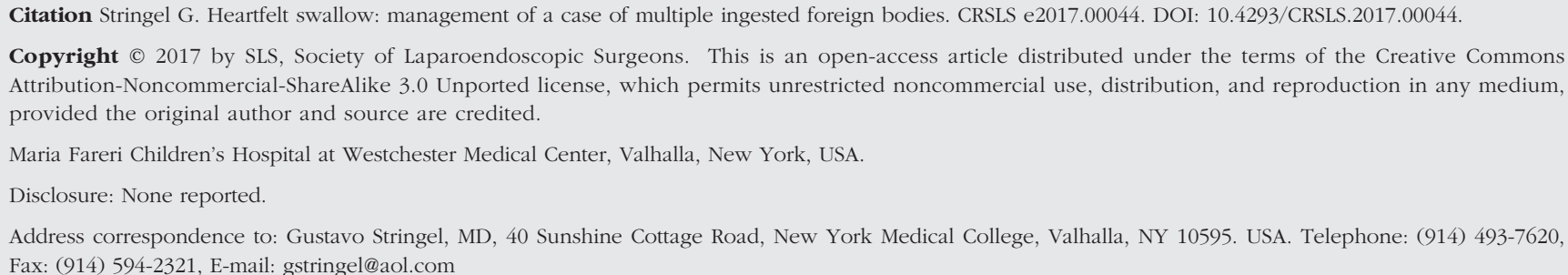

\section{INTRODUCTION}

Foreign bodies are ingested by individuals of all ages, but are most frequently encountered in pediatric patients. Of the 110,000 cases of ingested foreign objects reported to the American Association of Poison Control Centers in 2011, more than $85 \%$ occurred in the pediatric population. ${ }^{1}$ Reported data likely underestimates the number of pediatric cases, as up to $40 \%$ of foreign body ingestions in children are not witnessed, and up to $50 \%$ of children remain asymptomatic. Of the cases of foreign body ingestion that are brought to medical attention, $80 \%$ to $90 \%$ pass through the body naturally without complication or injury.1,2 The objects most commonly ingested by children, in descending order of frequency, are coins, magnets, batteries, small toys, jewelry, buttons, and bones. ${ }^{3}$ Sponge ingestion is an unusual presen- tation with potentially life- threatening consequences. Sponges are initially small enough to traverse the various sites of physiologic constriction within the proximal gastrointestinal tract. As the sponge migrates, it continues to absorb luminal contents, enlarge, and subsequently harden. In the bowel, enlarged sponges can cause obstruction. Patients present with symptoms of abdominal pain, loss of appetite, constipation, obstipation, and vomiting. Over time, the sponge hardens in the digestive tract and sharp edges develop, which put the bowel at significant risk of perforation. We report a complicated case of multiple foreign body ingestion in a child who required surgical intervention for suspected appendiceal and cecal inflammation or perforation secondary to an adjacent small lithium battery, but was found to have a normal appendix with a large obstructing sponge bezoar in the distal ileum. 
Heartfelt Swallow: Management of a Case of Multiple Ingested Foreign Bodies, Kirschenbaum MD et al.

\section{CASE REPORT}

A healthy 8-year-old male was brought to the ED with abdominal pain. His pain had started that morning, had a sharp quality, and was localized to the right lower quadrant. On further questioning, the patient admitted that he ingested a small lithium battery 1 week before. The patient denied nausea, emesis, fever, or chills. He had been eating and having normal bowel movements. Review of systems was otherwise unremarkable. His medical history was significant for lead poisoning at about age 3, which was treated with chelation therapy. He had no surgical or psychiatric history.

On physical examination, his abdomen was soft, mildly distended, and tender to palpation in the right lower quadrant. There was no rebound tenderness, guarding, or rigidity. Laboratory values were all within normal limits, with the exception of an elevated C-reactive protein of $7.50 \mathrm{mg} / \mathrm{dL}$, indicating inflammation. Computed tomography (CT) of the abdomen showed the battery located within or adjacent to the appendix, with concerns of appendiceal inflammation due to poorly defined regions of the appendix (Figures $\mathbf{1}$ and $\mathbf{2}$ ).

The patient was brought to the operating room for diagnostic laparoscopy because of worsening of his symptoms

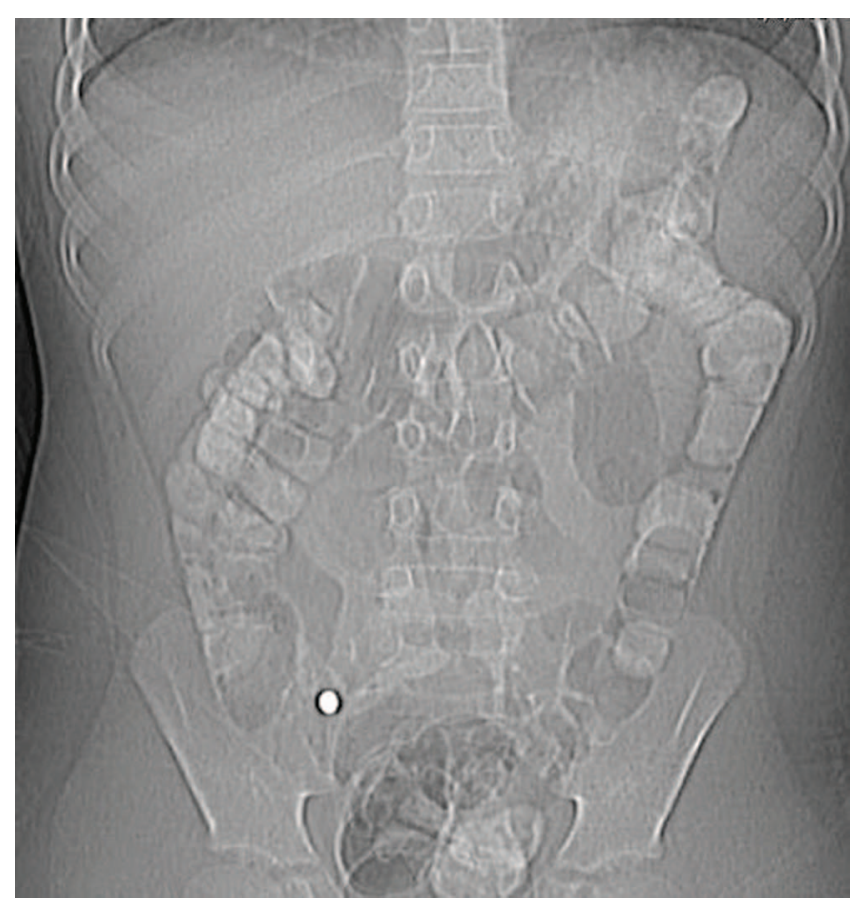

Figure 1. Lithium battery identified in the right lower quadrant of the abdomen.

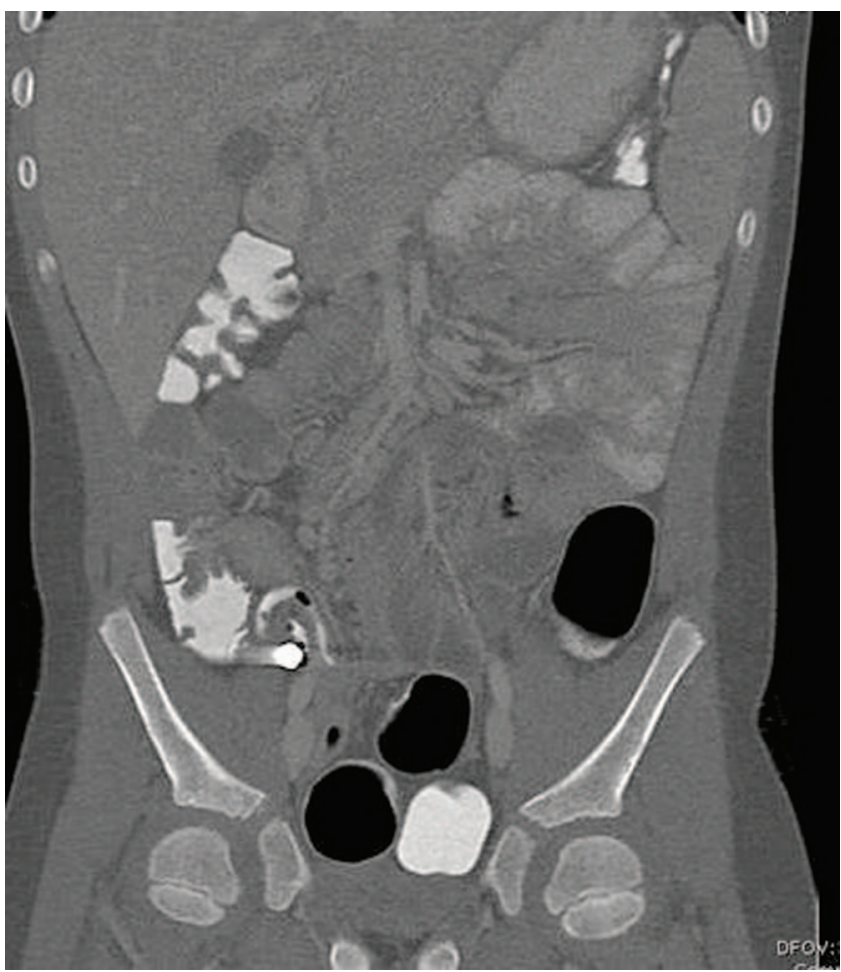

Figure 2. Battery identified near the appendix.

and increasing abdominal distention. The appendix was found to be intact and not inflamed, and no foreign body was found inside. A different large foreign body, which was not seen on preoperative imaging, was found stuck in the distal ileum, causing intestinal obstruction (Figure 3). The small bowel, cecum, and appendix were examined in an attempt to identify the battery seen on imaging, but

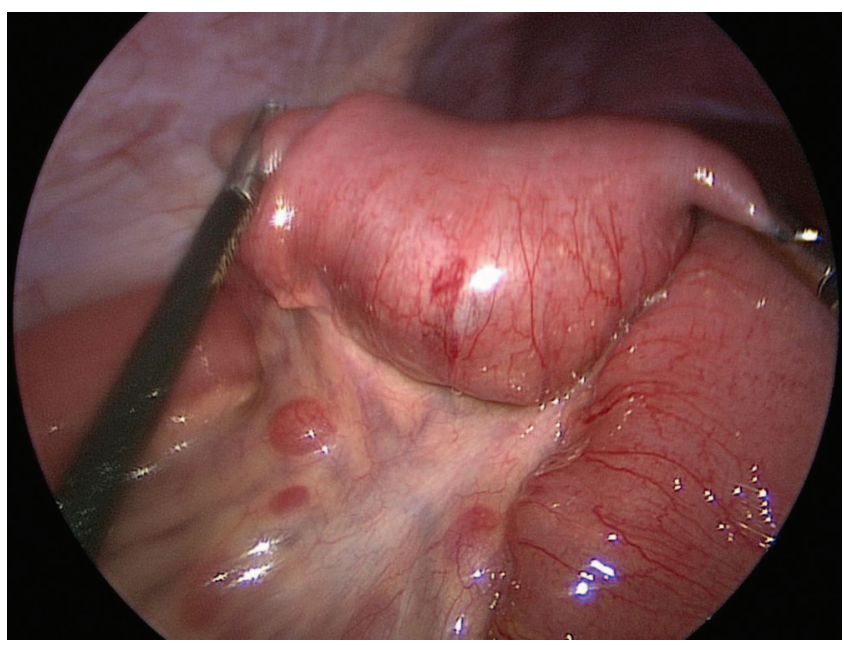

Figure 3. Ingested foreign body causing small bowel obstruction. 
there was difficulty in finding it. Fluoroscopy was implemented, and the battery was detected in the cecum. Proceeding first with extraction of the large foreign body in the distal ileum, a small McBurney's incision was made, and the small bowel was delivered outside of the peritoneal cavity. A heart-shaped foreign body could be distinguished through the bowel wall as the source of the intestinal obstruction (Figure 4). It had hardened, almost perforating the intestine. An enterotomy was made longitudinally, and the bezoar was extracted. It was identified as a heart-shaped polyurethane foam (PUF) sponge toy from an arts-and-crafts set for children (Figure 5). It measured $4.5 \times 3 \times 2 \mathrm{~cm}$.

The enterotomy was closed in a transverse fashion, and the bowel was irrigated to verify that there was no leakage. The intestine was returned to the peritoneal cavity, and the appendix was delivered out of it. An appendicostomy was made, and a 7-French Foley catheter was advanced into the appendix and the cecum. The balloon of the Foley catheter was inflated, and, under fluoroscopy, it was confirmed that it was distal to the battery. Attempts to milk the battery out were unsuccessful. Normal saline was flushed into the ascending colon, advancing the battery into the transverse colon. It was decided that the battery would most likely be evacuated naturally without complication. The Foley catheter was removed from the appendicostomy, and an appendectomy was performed.

In retrospect, after the surgery, we had delineated the heart-shaped object on the initial abdominal CT scan (Figure 6).

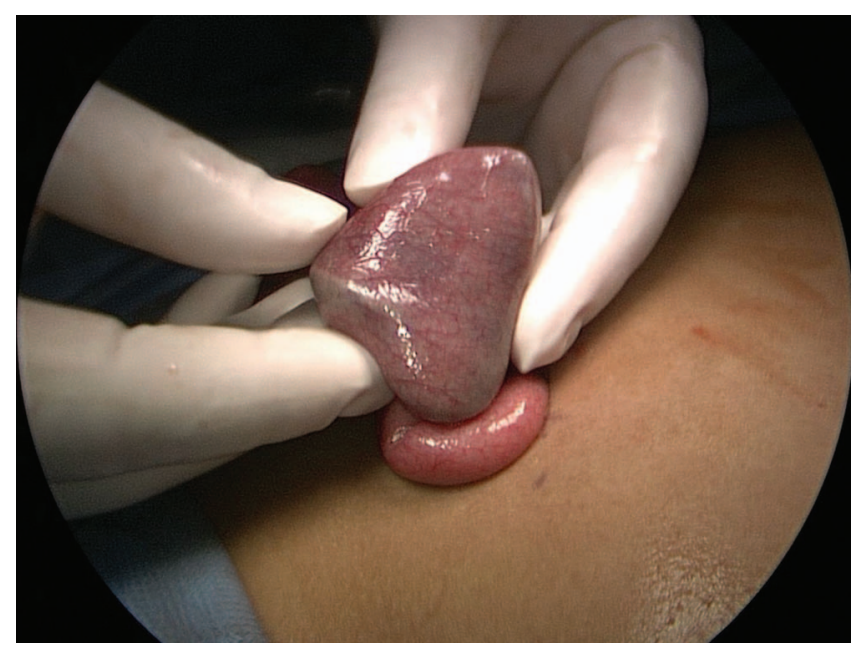

Figure 4. Heart-shaped foreign body seen through the intestinal wall.

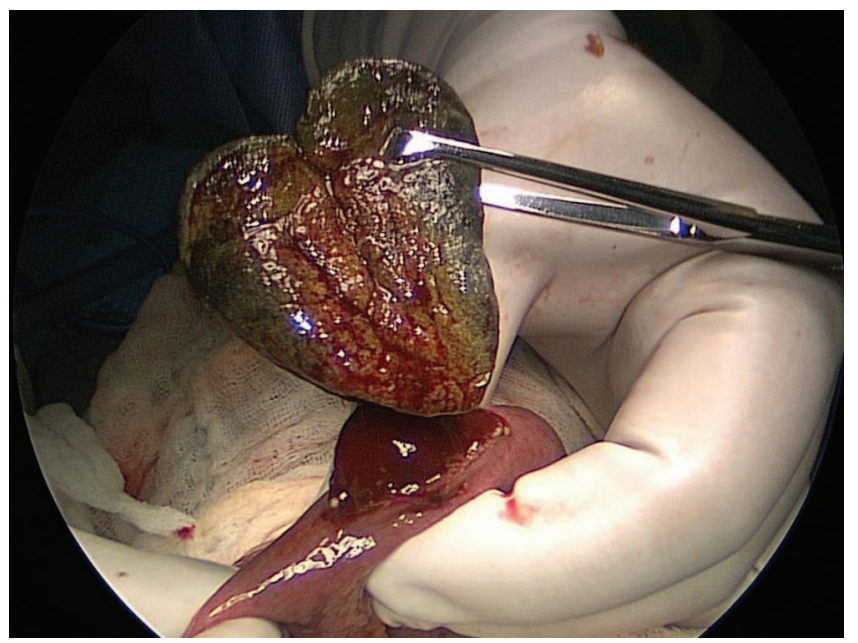

Figure 5. Heart-shaped PUF sponge extracted from the distal ileum.

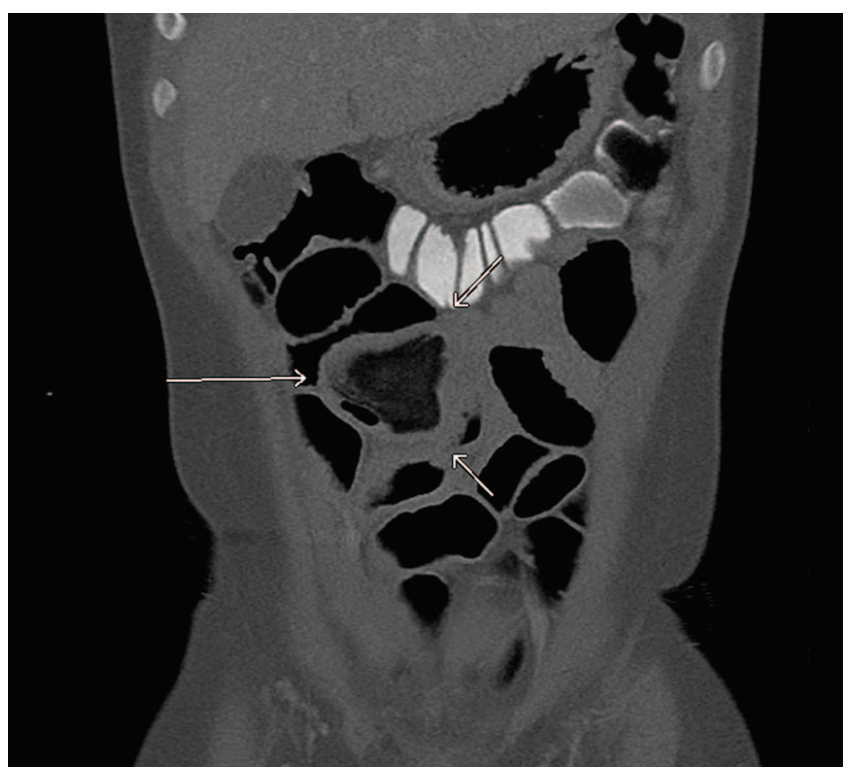

Figure 6. Heart-shaped object seen retrospectively on abdominal CT scan.

After surgery, an abdominal x-ray was performed to view the battery at baseline position. The patient was observed closely with daily abdominal radiographs to monitor the progress of the foreign body in the colon. On the third postoperative day, it became evident that the battery was failing to progress, so the patient was given a few doses of MiraLax (Schering-Plough HealthCare Products, Kenilworth, New Jersey, USA) and GoLytely (Braintree Laboratories, Braintree, Massachusetts, USA) to facilitate the process. The battery still did not advance. Pediatric gastroenterology was consulted, and the patient underwent 
colonoscopy 1 week after surgery. The battery was visualized in the colon, but could not be retrieved safely. The patient underwent a second colonoscopy the following day, and the battery was finally retrieved. It was small, measuring $5 \mathrm{~mm}$ in diameter, and was firmly attached to the wall of the ascending colon. The patient tolerated the procedures well. He was discharged home the following day on a regular diet. He was doing well on follow-up 4 weeks later.

\section{DISCUSSION}

PUF is a compressible material made from hydrophobic and hydrophilic fibers mixed with super absorbent polymers and other additives in various ratios. It is best known for its applications in furniture and cars, but has been gaining popularity in the medical arena. ${ }^{4}$ PUF's ability to retain and absorb large amounts of fluid makes it an attractive material for a surgical sponge, but a very dangerous material for a child to ingest.

Ingested foreign bodies usually pass through the digestive tract without complication or injury. ${ }^{1,2}$ If obstruction occurs, it is usually in the cricopharyngeal area, middle one-third of the esophagus, lower esophageal sphincter, pylorus, or ileocecal valve. ${ }^{2}$ An ingested PUF sponge is unique, in that it is initially small enough to traverse the various sites of physiologic constriction within the upper gastrointestinal tract. Because PUF is highly absorbent, it retains large quantities of gastrointestinal contents, and will expand until it becomes large enough to cause obstruction. There is significant potential for obstruction in the distal ileum, as evidenced in our patient. The clinical presentation of a PUF sponge bezoar is typically that of intestinal obstruction with abdominal pain, loss of appetite, constipation, obstipation, and vomiting. As the density of the sponge increases, it also begins to harden. Sharp edges will develop in the initially smooth object, putting the bowel at significant risk of perforation. The progression from smooth to sharp borders, along with the associated gastrointestinal complications, is similar to those reported with vinyl glove ingestion. ${ }^{5}$

Preoperative imaging that could assist in identifying areas of obstruction after PUF ingestion was helpful only retrospectively in our case ${ }^{6,7}$ (Figure 6).

Preoperative imaging enabled clear visualization of the radiopaque lithium battery located within or adjacent to the appendix, with concern about appendiceal or cecal inflammation or perforation.
The PUF sponge was removed surgically without complication, whereas multiple interventions were required to ensure the safe removal of the ingested battery. Removal of the battery was challenging, as prolonged alkaline caustic injury allowed for localized tissue liquefaction and adherence to the bowel mucosa.

Cases of PUF ingestion in pediatric patients have been described in the literature, with development of similar gastrointestinal complications of obstruction and perforation. ${ }^{6-8}$ However, in all of the cases the ingested PUF was taken from upholstery, which included cushioning from a settee, car seat, sofa, and carpet. Several cases of significant pediatric morbidity and 1 case of mortality have also been reported because of ingestion of beads or balls of superabsorbent polymers that were used for agricultural products, decorative items, and children's toys. ${ }^{9-12}$ Our study documents an unusual intestinal obstruction secondary to ingestion of a heart-shaped PUF sponge toy from a children's arts-and-crafts set. In addition, our patient's sponge bezoar was complicated by the presence of a different radiopaque ingested foreign body, which required multiple attempts for safe retrieval. Our patient did not demonstrate an association between the sponge ingestion and anemia, as described in earlier cases. ${ }^{6,7}$

\section{CONCLUSION}

This case illustrates complications that may arise in the retrieval of multiple ingested foreign bodies from the gastrointestinal tract. A large obstructing PUF sponge bezoar that was not visualized on preoperative imaging was found in our patient, who was undergoing laparoscopy for suspected appendiceal or cecal inflammation or perforation secondary to an ingested lithium battery. The PUF sponge bezoar was removed from the small intestine via laparoscopy-assisted enterotomy. The battery was removed from the colon via colonoscopy.

\section{References:}

1. Wright CC, Closson FT. Updates in pediatric gastrointestinal foreign bodies. Pediatr Clin North Am. 2013;60:1221-1239.

2. Uyemura MC. Foreign body ingestion in children. Am Fam Physician. 2005;72:287-291.

3. Pugmire BS, Lim R, Avery LL. Review of ingested and aspirated foreign bodies in children and their clinical significance for radiologists. Radiographics. 2015;35:1528-1538. 
4. Thavarajah R, Joshua E, Rao UK, Ranganathan K. On factors influencing the absorption capacity of surgical sponges. J Maxillofac Oral Surg. 2014;13:238-243.

5. Stringel G, Parker M, McCoy E. Vinyl glove ingestion in children: a word of caution. J Pediatr Surg. 2012;47:996-998.

6. Allen NM, O’Donovan DJ. Foam eater. J Pediatr. 2007;151: 710.

7. Gupta A, Rajput S, Maduabuchi G, Kumar P. Sponge eating: is it an obsessive compulsive disorder or an unusual form of pica? Acta Paediatr. 2007;96:1853-1854.

8. McAlinden MG, Potts SR. Sponge bezoar: a rare cause of abdominal pain. Ulster Med J. 1999;68:36-37.
9. Moon JS, Bliss D, Hunter CJ. An unusual case of small bowel obstruction in a child caused by ingestion of water-storing gel beads. J Pediatr Surg. 2012;47:E19-E22.

10. Mirza B, Ijaz L, Sheikh A. Decorative crystal balls causing intestinal perforation. J Indian Assoc Pediatr Surg. 2011;16:106107.

11. Mirza B, Sheikh A. Mortality in a case of crystal gel ball ingestion: an alert for parents. APSP J Case Rep. 2012;3:6.

12. Zamora IJ, Vu LT, Larimer EL, Olutoye OO. Water-absorbing balls: a "growing" problem. Pediatrics. 2012;130:e1011-e1014. 